\title{
MINIMAL CONVEX FUNCTIONS BOUNDED BELOW BY THE DUALITY PRODUCT
}

\author{
J.-E. MARTÍNEZ-LEGAZ AND B. F. SVAITER
}

(Communicated by Jonathan M. Borwein)

\begin{abstract}
It is well known that the Fitzpatrick function of a maximal monotone operator is minimal in the class of convex functions bounded below by the duality product. Our main result establishes that, in the setting of reflexive Banach spaces, the converse also holds; that is, every such minimal function is the Fitzpatrick function of some maximal monotone operator. Whether this converse also holds in a nonreflexive Banach space remains an open problem.
\end{abstract}

\section{INTRODUCTION}

Convex analysis is involved in many branches of mathematics, from functional analysis to optimization. Maximal monotone operators appear naturally in convex analysis, but their study is in many parts performed outside convex analysis. This statement may undergo a drastic change. In a 1988 paper, Fitzpatrick [4] proved that any maximal monotone operator can be represented by convex functions in a special class. Moreover, he explicitly defined a convex representation of a given maximal monotone operator and proved this function to be minimal in the class. These results were recently rediscovered [6, 2], and since then Fitzpatrick's results have been the subject of intense research $[9,3,12,7,10,5,11,8,1,13]$.

The aim of this paper is to provide a partial converse to one of Fitzpatrick's results. Namely, we will provide a partial characterization of the minimal elements of the family of convex functions bounded below by the duality product. In the special setting of a reflexive Banach space, we will show that the minimal elements are the Fitzpatrick functions associated to maximal monotone operators.

This paper is organized as follows. In Section 2 we introduce the notation and some basic definitions and recall some auxiliary results. In Section 3 the main theorem is proved and some open questions are discussed.

Received by the editors June 20, 2006.

2000 Mathematics Subject Classification. Primary 47H05; Secondary 52A41, 26B25.

The first author was partially supported by the Ministerio de Ciencia y Tecnología, Project MTM2005-08572-C03-03. He also thanks the support of the Barcelona Economics Program of CREA.

The second author was partially suported by CNPq grant n. 300755/2005-8 and Edital Universal 476842/03-2.

This work was initiated during a visit of the second author to the Universitat Autònoma de Barcelona in March 2006. 


\section{BASIC DEFINITIONS AND AUXILIARY RESUlTS}

Let $X$ be a real Banach space and $X^{*}$ be its dual. For $x \in X, x^{*} \in X^{*}$ we use the notation

$$
\left\langle x, x^{*}\right\rangle=x^{*}(x) .
$$

We will identify a point-to-set operator $A: X \rightrightarrows X^{*}$ with its graph,

$$
A \triangleq\left\{\left(x, x^{*}\right) \mid x^{*} \in A(x)\right\} .
$$

The operator $A: X \rightrightarrows X^{*}$ is monotone if

$$
\left\langle x-y, x^{*}-y^{*}\right\rangle \geq 0, \quad \forall\left(x, x^{*}\right),\left(y, y^{*}\right) \in A .
$$

A monotone operator is maximal monotone if it is not properly contained in any other monotone operator.

The conjugate of $f: X \rightarrow \bar{R}$ is $f^{*}: X^{*} \rightarrow \bar{R}$,

$$
f^{*}\left(x^{*}\right):=\sup _{x \in X}\left\langle x, x^{*}\right\rangle-f(x) .
$$

If $f$ is an (extended) real valued function defined on $X \times X^{*}$, then $f^{*}$ is defined on $X^{*} \times X^{* *}$. We will identify an element $x \in X$ with its image under the canonical injection of $X$ into $X^{* *}$,

$$
x: X^{*} \rightarrow R, \quad x\left(x^{*}\right)=x^{*}(x)=\left\langle x, x^{*}\right\rangle .
$$

We use this convention whenever we write $f^{*}\left(x^{*}, x\right)$ for $f: X \times X^{*} \rightarrow \bar{R}, x \in X$ and $x^{*} \in X^{*}$.

The Fitzpatrick function associated to an operator $A: X \rightrightarrows X^{*}$ is defined as

$$
\begin{aligned}
\varphi_{A}\left(x, x^{*}\right) & =\sup _{\left(y, y^{*}\right) \in A}\left\langle x-y, y^{*}-x^{*}\right\rangle+\left\langle x, x^{*}\right\rangle \\
& =\sup _{\left(y, y^{*}\right) \in A}\left\langle x, y^{*}\right\rangle+\left\langle y, x^{*}\right\rangle-\left\langle y, y^{*}\right\rangle .
\end{aligned}
$$

This function has some nice properties. It is convex and l.s.c., and if $A$ is maximal monotone, then $\varphi_{A}$ characterizes $A$ :

Theorem 1 ([4, Cor. 3.9, Prop. 3.2 and Thm. 3.7]). Let $A \subset X \times X^{*}$ be maximal monotone. Then, for any $\left(x, x^{*}\right) \in X \times X^{*}$,

$$
\begin{aligned}
& \varphi_{A}\left(x, x^{*}\right) \geq\left\langle x, x^{*}\right\rangle \\
& \varphi_{A}\left(x, x^{*}\right)=\left\langle x, x^{*}\right\rangle \Longleftrightarrow\left(x, x^{*}\right) \in A
\end{aligned}
$$

and

$$
\begin{aligned}
& \left(\varphi_{A}\right)^{*}\left(x^{*}, x\right) \geq\left\langle x, x^{*}\right\rangle \\
& \left(\varphi_{A}\right)^{*}\left(x^{*}, x\right)=\left\langle x, x^{*}\right\rangle \Longleftrightarrow\left(x, x^{*}\right) \in A .
\end{aligned}
$$

Moreover, $\varphi_{A}$ is minimal in the family of convex functions defined on $X \times X^{*}$ that are bounded below by the duality product.

Proof. The only part of the statement that cannot be found in [4] is the minimality of $\varphi_{A}$, though it easily follows from the main results of that paper. Indeed, suppose that $f: X \times X^{*} \rightarrow \bar{R}$ is convex, bounded below by the duality product and $f \leq \varphi_{A}$; then, by $(1), f\left(x, x^{*}\right)=\left\langle x, x^{*}\right\rangle$ for all $\left(x, x^{*}\right) \in A$ and hence, in view of $[4$, Thm. 3.7], $\varphi_{A} \leq f$. 
Let us denote by $\pi$ the duality product,

$$
\pi: X \times X^{*} \rightarrow R, \quad \pi\left(x, x^{*}\right)=\left\langle x, x^{*}\right\rangle,
$$

and let $\mathcal{F}$ be the family of convex functions on $X \times X^{*}$ that are bounded below by the duality product,

$$
\mathcal{F}=\left\{f: X \times X^{*} \rightarrow \bar{R} \mid f \text { is convex and proper, } f \geq \pi\right\} .
$$

As we already mentioned, Fitzpatrick functions of maximal monotone operators are minimal in the family $\mathcal{F}$. It is natural to ask which are the minimal elements of this family. We will partially characterize the minimal elements of $\mathcal{F}$ in a generic Banach space, and for reflexive spaces we will show that these minimal elements are Fitzpatrick functions.

The following auxiliary result of Burachik and Svaiter [3] will be needed:

Theorem 2 ([3, Thm. 3.1]). Let $X$ be reflexive and $h: X \times X^{*} \rightarrow \bar{R}$ be a lower semicontinuous convex function. Define

$$
A:=\left\{\left(x, x^{*}\right) \in X \times X^{*} \mid h\left(x, x^{*}\right)=\left\langle x, x^{*}\right\rangle\right\} .
$$

If

$$
h\left(x, x^{*}\right) \geq\left\langle x, x^{*}\right\rangle, \quad h^{*}\left(x^{*}, x\right) \geq\left\langle x, x^{*}\right\rangle, \quad \forall\left(x, x^{*}\right) \in X \times X^{*},
$$

then $A$ is maximal monotone and

$$
A=\left\{\left(x, x^{*}\right) \in X \times X^{*} \mid h^{*}\left(x^{*}, x\right)=\left\langle x, x^{*}\right\rangle\right\} .
$$

\section{MAin RESUlts}

The following lemma will be useful to prove the main result:

Lemma 3. For any function $f: X \times X^{*} \rightarrow \bar{R}$ and any $\left(x, x^{*}\right),\left(y, y^{*}\right) \in X \times X^{*}$, $p, q \geq 0, p+q=1$, it holds that

$$
p \max \left\{f\left(x, x^{*}\right),\left\langle x, x^{*}\right\rangle\right\}+q \max \left\{f^{*}\left(y^{*}, y\right),\left\langle y, y^{*}\right\rangle\right\} \geq\left\langle p x+q y, p x^{*}+q y^{*}\right\rangle .
$$

Proof. By direct algebraic manipulation, using Fenchel inequality one gets

$$
\begin{aligned}
\left\langle p x+q y, p x^{*}+q y^{*}\right\rangle & =p^{2}\left\langle x, x^{*}\right\rangle+p q\left(\left\langle x, y^{*}\right\rangle+\left\langle y, x^{*}\right\rangle\right)+q^{2}\left\langle y, y^{*}\right\rangle \\
& \leq p^{2}\left\langle x, x^{*}\right\rangle+p q\left(f\left(x, x^{*}\right)+f^{*}\left(y^{*}, y\right)\right)+q^{2}\left\langle y, y^{*}\right\rangle \\
& =p\left(p\left\langle x, x^{*}\right\rangle+q f\left(x, x^{*}\right)\right)+q\left(p f^{*}\left(y^{*}, y\right)+q\left\langle y, y^{*}\right\rangle\right) \\
& \leq p \max \left\{f\left(x, x^{*}\right),\left\langle x, x^{*}\right\rangle\right\}+q \max \left\{f^{*}\left(y^{*}, y\right),\left\langle y, y^{*}\right\rangle\right\},
\end{aligned}
$$

which is the desired result.

Corollary 4. For any $f \in \mathcal{F}$ and $\left(x, x^{*}\right) \in X \times X^{*}$ there exists $h \in \mathcal{F}$ such that

$$
f \geq h, \quad \max \left\{f^{*}\left(x^{*}, x\right),\left\langle x, x^{*}\right\rangle\right\} \geq h\left(x, x^{*}\right) .
$$

Proof. Define

$$
h=\mathrm{conv} \min \left\{f, \delta_{\left\{\left(x, x^{*}\right)\right\}}+\max \left\{f^{*}\left(x^{*}, x\right),\left\langle x, x^{*}\right\rangle\right\}\right\},
$$

the largest convex minorant of $f$ and $\delta_{\left\{\left(x, x^{*}\right)\right\}}+\max \left\{f^{*}\left(x^{*}, x\right),\left\langle x, x^{*}\right\rangle\right\}$, with $\delta$ denoting an indicator function in the sense of convex analysis. Since these two functions are convex, one has

$$
h\left(y, y^{*}\right)=\inf \left\{p f\left(z, z^{*}\right)+q \max \left\{f^{*}\left(x^{*}, x\right),\left\langle x, x^{*}\right\rangle\right\}\right\}, \quad \forall\left(y, y^{*}\right) \in X \times X^{*},
$$


the infimum being over all $p, q \geq 0$ and $\left(z, z^{*}\right) \in X \times X^{*}$ such that

$$
p+q=1, \quad p\left(z, z^{*}\right)+q\left(x^{*}, x\right)=\left(y, y^{*}\right) .
$$

Hence, by Lemma $3, h \geq \pi$. From the definition of $h$ it follows that it satisfies the required conditions.

Theorem 5. For any $f \in \mathcal{F}$ there exists a minimal $g \in \mathcal{F}$ such that $f \geq g$. Moreover, any minimal element $g$ of $\mathcal{F}$ is l.s.c., satisfies

$$
g^{*}\left(x^{*}, x\right) \geq g\left(x, x^{*}\right), \quad \forall\left(x, x^{*}\right) \in X \times X^{*},
$$

and, if $X$ is reflexive, then $g=\varphi_{A}$ for some maximal monotone $A: X \rightrightarrows X^{*}$.

Proof. The family $\mathcal{F}$ is (decreasingly) inductively ordered. Indeed, if $\left\{f_{\alpha}\right\}_{\alpha \in \Lambda}$ is a totally ordered family of functions of $\mathcal{F}$, defining

$$
\hat{f}=\inf _{\alpha \in \Lambda} f_{\alpha},
$$

we have $\hat{f}$ convex, $\hat{f} \geq \pi$ and $f_{\alpha} \geq \hat{f}$ for any $\alpha \in \Lambda$. Hence, using Zorn's Lemma we conclude that given any $f \in \mathcal{F}$ there exists a minimal $g \in \mathcal{F}, f \geq g$.

Now, let $g \in \mathcal{F}$ be a minimal element of $\mathcal{F}$ and let $\mathrm{cl} g$ be the lower semicontinuous closure of $g$. Since $g$ is convex, $g \geq \pi$ and the duality product is continuous, it follows that the lower semicontinuous closure of $g$ is also convex and greater than $\pi$, that is, $\operatorname{cl} g \in \mathcal{F}$. Hence, $g$ being minimal, it must be equal to its lower semicontinuous closure, i.e., $g$ is lower semicontinuous. Take an arbitrary $\left(x, x^{*}\right) \in$ $X \times X^{*}$. If $g^{*}\left(x^{*}, x\right)=\infty$, then, trivially, $g\left(x, x^{*}\right) \leq g^{*}\left(x^{*}, x\right)$. So, assume that $g^{*}\left(x^{*}, x\right)<\infty$. By Corollary 4 , there exists $h \in \mathcal{F}$ such that $g \geq h$ and $h\left(x, x^{*}\right) \leq$ $\max \left\{g^{*}\left(x^{*}, x\right),\left\langle x, x^{*}\right\rangle\right\}$. Since $g$ is minimal, we actually have $h=g$ and hence

$$
g\left(x, x^{*}\right) \leq \max \left\{g^{*}\left(x^{*}, x\right),\left\langle x, x^{*}\right\rangle\right\} .
$$

In particular, $g\left(x, x^{*}\right)$ is also finite. Using Fenchel inequality, we obtain

$$
\max \left\{g^{*}\left(x^{*}, x\right),\left\langle x, x^{*}\right\rangle\right\} \leq \max \left\{g^{*}\left(x^{*}, x\right), \frac{1}{2}\left(g\left(x, x^{*}\right)+g^{*}\left(x^{*}, x\right)\right)\right\},
$$

which, combined with the previous inequality, implies that $g\left(x, x^{*}\right) \leq g^{*}\left(x^{*}, x\right)$.

If $X$ is reflexive, then, applying Theorem 2, we conclude that the set

$$
A=\left\{\left(x, x^{*}\right) \in X \times X^{*}: g\left(x, x^{*}\right)=\left\langle x, x^{*}\right\rangle\right\}
$$

is maximal monotone. Hence $\varphi_{A} \in \mathcal{F}$ and, applying Theorem 1 , we deduce that $g \geq \varphi_{A}$, which, as $g$ is minimal, implies $g=\varphi_{A}$.

Using Lemma 3 we also obtain the following result.

Proposition 6. For any $f \in \mathcal{F}$ such that

$$
f^{*}\left(x^{*}, x\right) \geq\left\langle x, x^{*}\right\rangle, \quad \forall\left(x, x^{*}\right) \in X \times X^{*},
$$

there exists $h \in \mathcal{F}$ such that

$$
\min \left\{f\left(x, x^{*}\right), f^{*}\left(x^{*}, x\right)\right\} \geq h\left(x, x^{*}\right), \quad \forall\left(x, x^{*}\right) \in X \times X^{*} .
$$

Proof. For $\left(x, x^{*}\right) \in X \times X^{*}$ define $h\left(x, x^{*}\right)=\inf \left\{p f\left(y, y^{*}\right)+q f^{*}\left(z^{*}, z\right)\right\}$, the infimum being over all $p, q \geq 0$ and $\left(y, y^{*}\right),\left(z, z^{*}\right) \in X \times X^{*}$ such that

$$
p+q=1, \quad p\left(y, y^{*}\right)+q\left(z, z^{*}\right)=\left(x, x^{*}\right) .
$$


Clearly the function $h: X \times X^{*} \rightarrow \bar{R}$ so defined satisfies (3); in fact, since $f$ and $f^{*}$ are convex, it is the largest convex minorant of $f$ satisfying this inequality. Moreover, by Lemma $3, h \geq \pi$, so that $h \in \mathcal{F}$.

Since the duality product is continuous on $X \times X^{*}$, Proposition 6 still holds replacing $\mathcal{F}$ by $\mathcal{F}_{0}$

$$
\mathcal{F}_{0}=\{f \in \mathcal{F}: f \text { l.s.c. }\} \text {. }
$$

Moreover, from Theorem 5 one concludes that the family $\mathcal{F}_{0}$ is also inductively ordered.

Since Fitzpatrick functions are not only lower semicontinuous in the strong topology of the space $X \times X^{*}$ but also in the $w \times w^{*}$ topology, that is, in the weak topology on $X \times X^{*}$ determined by the functionals in $X^{*} \times X$, we will next consider the family

$$
\mathcal{F}_{0 w}=\left\{f \in \mathcal{F}: f \text { l.s.c. in the } w \times w^{*} \text { topology }\right\} .
$$

If $X$ is reflexive, the $w \times w^{*}$ topology of $X \times X^{*}$ coincides with the weak topology. In this case, for convex functions, lower semicontinuity (in the strong topology) is equivalent to lower semicontinuity in the $w \times w^{*}$ topology, and then $\mathcal{F}_{0 w}=\mathcal{F}_{0}$.

If $X$ is non-reflexive, the preceding reasoning fails and the inclusion

$$
\mathcal{F}_{0 w} \subset \mathcal{F}_{0}
$$

may be strict. Moreover, in infinite dimensional Banach spaces the duality product is not continuous in the $w \times w^{*}$ topology of $X \times X^{*}$. Even though, Fitzpatrick functions of maximal monotone operators belong to $\mathcal{F}_{0 w}$. This discussion raises some questions for the case where $X$ is non-reflexive:

1) Is the family $\mathcal{F}_{0 w}$ inductively ordered?

2) Does Proposition 6 hold replacing $\mathcal{F}$ by $\mathcal{F}_{0 w}$ ?

Finally, a full characterization of the minimal elements of $\mathcal{F}$ and $\mathcal{F}_{0 w}$ is missing in the non-reflexive case.

\section{REFERENCES}

[1] R. S. Burachik and S. Fitzpatrick. On a family of convex functions associated to subdifferentials. J. Nonlinear Convex Anal., 6(1):165-171, 2005. MR2138108 (2006b:47081)

[2] R. S. Burachik and B. F. Svaiter. Maximal monotone operators, convex functions and a special family of enlargements. Set-Valued Anal., 10(4):297-316, 2002. MR1934748 (2003k:90085)

[3] R. S. Burachik and B. F. Svaiter. Maximal monotonicity, conjugation and the duality product. Proc. Amer. Math. Soc., 131(8):2379-2383 (electronic), 2003. MR1974634 (2004a:49037)

[4] S. Fitzpatrick. Representing monotone operators by convex functions. In Workshop/Miniconference on Functional Analysis and Optimization (Canberra, 1988), volume 20 of Proc. Centre Math. Anal. Austral. Nat. Univ., pages 59-65. Austral. Nat. Univ., Canberra, 1988. MR1009594 (90i:47054)

[5] J.-E. Martínez-Legaz and B. F. Svaiter. Monotone operators representable by l.s.c. convex functions. Set-Valued Anal., 13(1):21-46, 2005. MR2128696 (2005m:47104)

[6] J.-E. Martínez-Legaz and M. Théra. A convex representation of maximal monotone operators. J. Nonlinear Convex Anal., 2(2):243-247, 2001. Special issue for Professor Ky Fan. MR1848704 (2002e:49035)

[7] J.-P. Penot. The relevance of convex analysis for the study of monotonicity. Nonlinear Anal., 58(7-8):855-871, 2004. MR2086060 (2005g:49026)

[8] S. Reich and S. Simons. Fenchel duality, Fitzpatrick functions and the Kirszbraun-Valentine extension theorem. Proc. Amer. Math. Soc., 133(9):2657-2660 (electronic), 2005. MR2146211 (2006d:46025)

[9] J. P. Revalski and M. Théra. Enlargements and sums of monotone operators. Nonlinear Anal., 48(4, Ser. A: Theory Methods):505-519, 2002. MR1871464 (2002k:47104) 
[10] S. Simons and C. Zălinescu. A new proof for Rockafellar's characterization of maximal monotone operators. Proc. Amer. Math. Soc., 132(10):2969-2972 (electronic), 2004. MR2063117 (2005f:47121)

[11] S. Simons and C. Zălinescu. Fenchel duality, Fitzpatrick functions and maximal monotonicity. J. Nonlinear Convex Anal., 6(1):1-22, 2005. MR2138099 (2005k:49102)

[12] B. F. Svaiter. Fixed points in the family of convex representations of a maximal monotone operator. Proc. Amer. Math. Soc., 131(12):3851-3859 (electronic), 2003. MR1999934 (2004h:49016)

[13] C. Zălinescu. A new proof of the maximal monotonicity of the sum using the Fitzpatrick function. In Variational analysis and applications, volume 79 of Nonconvex Optim. Appl., pages 1159-1172. Springer, New York, 2005.

Departament d'Economia i D'Història Econòmica, Universitat Autònoma de BarceLONA, 08193 BellaterRA, SpAin

E-mail address: JuanEnrique.Martinez@uab.es

Instituto de Matemática Pura e Aplicada (IMPA), Estrada Dona Castorinha 110, Jardim BotÂnico, Rio de JANeiro, CEP 22460-320, Brazil

E-mail address: benar@impa.br 